\title{
Realistic two-baryon potential coupling two-nucleon and nucleon- $\Delta$-isobar states: Fit and applications to three-nucleon system
}

\author{
A. Deltuva, ${ }^{1, *}$ R. Machleidt, ${ }^{2}$ and P. U. Sauer ${ }^{1}$ \\ ${ }^{1}$ Institut für Theoretische Physik, Universität Hannover, D-30167 Hannover, Germany \\ ${ }^{2}$ Department of Physics, University of Idaho, Moscow, Idaho 83844, USA
}

(Received 25 April 2003; published 28 August 2003)

\begin{abstract}
A two-baryon coupled-channel potential is developed. It couples two-nucleon states and states in which one nucleon is turned into a $\Delta$ isobar. It is developed as extension of the purely nucleonic charge-dependent (CD) Bonn potential. It is fitted to two-nucleon scattering data up to $350 \mathrm{MeV}$ nucleon lab energy. Since scattering energies just touch the pion-production threshold, the $\Delta$ isobar is considered a stable baryon. The resulting fit of the coupled-channel potential is of the same quality as that for CD Bonn. The coupled-channel potential is as realistic as CD Bonn and as any other modern two-nucleon potential. It is charge-dependent as CD Bonn. It is employed for the description of elastic and inelastic three-nucleon scattering. The $\Delta$ isobar yields an effective three-nucleon force in the three-nucleon system, besides other effects. $\Delta$-isobar effects in threenucleon scattering are isolated and discussed.
\end{abstract}

DOI: 10.1103/PhysRevC.68.024005

PACS number(s): 21.45.+v, 21.30.-x, 24.70.+s, 25.10.+s

\section{INTRODUCTION}

The properties of the three-nucleon bound state were described by us in Ref. [1], elastic nucleon-deuteron scattering and breakup in Refs. [2-5] and inelastic electromagnetic (em) reactions of the three-nucleon system in Ref. [6]. The description was in terms of a two-baryon coupled-channel potential and of a corresponding e.m. current. The channels coupled are purely nucleonic ones and those in which one nucleon $(N)$ is turned into a $\Delta$ isobar.

We like to take the excitation of a $\Delta$ isobar explicitly into account: The $\Delta$ isobar yields an effective three-nucleon force, it effectively contributes to the electroweak exchange current, and it provides, in principle, a mechanism for pion production and absorption. Since the inelasticities of twonucleon scattering remain very small in isospin singlet twonucleon partial waves up to about $500 \mathrm{MeV}$ center of mass (c.m.) energy and since the inelasticities in the isospin triplet partial waves are in the same energy regime mostly due to single-pion production, though energies may well be above two-pion threshold, the inelastic two-baryon channels are assumed to have single $\Delta$-isobar excitation at most [7]. A noncovariant Hamiltonian with a two-baryon coupled-channel potential allowing the $\Delta$ isobar further coupling to pionnucleon states can therefore provide a common unifying basis [8] for nuclear phenomena at low and intermediate energies. However, we confine the description at present to processes below the pion-production threshold. We therefore omit in this paper, as in Refs. [2-6], the coupling of the $\Delta$ isobar to pion-nucleon states and consider the $\Delta$ isobar a stable baryon of mass $1232 \mathrm{MeV}$ with spin and isospin $\frac{3}{2}$.

Reference [5] developed a new technique for solving three-particle equations; it is based on the Chebyshev expan-

\footnotetext{
*On leave from Institute of Theoretical Physics and Astronomy, Vilnius University, Vilnius 2600, Lithuania. Electronic address: deltuva@itp.uni-hannover.de
}

sion of the two-baryon transition matrix; the Chebyshev expansion is systematic and efficient. The new technique replaces our old one requiring a separable expansion of the two-baryon transition matrix; though also quite reliable, the need for a separable expansion made the old technique rather inflexible to us in applications. In contrast, the new one allows us to use directly any two-nucleon potential and any coupled-channel extension of theirs as dynamic input for the description of the three-nucleon bound state and of threenucleon scattering. But, whereas we used the most modern two-nucleon potentials as reference [9-11], our construction of the coupled-channel extension [1] has been a rather old method till now. It ensures phase equivalence with the nucleonic reference potential at zero two-nucleon kinetic energy; phase inequivalence increases with increasing two-nucleon scattering energy [12]. That fact has been known for long, but was considered tolerable by us as long as only the properties of the three-nucleon bound state and lowenergy three-nucleon scattering were in focus. However, that phase inequivalence becomes unacceptable, once threenucleon scattering at moderate energies up to the pionproduction threshold is discussed. This paper repairs the coupled-channel part of the dynamic input for our description of the three-nucleon bound state and of three-nucleon scattering.

Section II reports our fit of a new realistic coupledchannel potential; it is constructed as an extension of the charge-dependent $(\mathrm{CD})$ Bonn potential. Based on that new coupled-channel potential, Sec. III gives results for the threenucleon bound state. Section IV gives selected results for elastic nucleon-deuteron scattering and for breakup. Section $\mathrm{V}$ compares the $\Delta$-isobar effects previously obtained to those of this paper. Section VI contains a summary and our conclusions.

\section{FIT OF NEW REALISTIC COUPLED-CHANNEI POTENTIAL}

The two-baryon coupled-channel potential is graphically defined in Fig. 1; it has a transition potential from nucleonic 


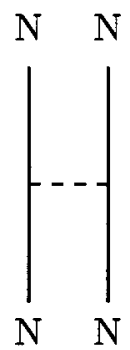

(a)

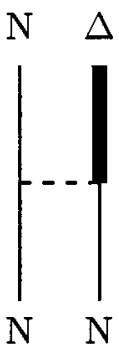

(b)

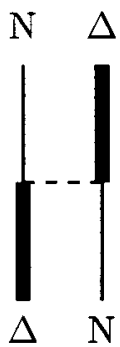

(c)

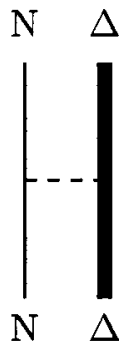

(d)
FIG. 1. Two-baryon coupled-channel potential. A thin vertical line denotes a nucleon, a thick vertical line a $\Delta$ isobar, and a dashed horizontal line the instantaneous potential. The Hermitian conjugate of the transition process (b) is not shown.

to the nucleon- $\Delta$ states and a direct potential between the latter ones; it acts in isospin triplet partial waves only; in isospin singlet partial waves it is purely nucleonic. The coupled-channel potential provides additional attraction between two nucleons by the virtual excitation of a nucleon to a $\Delta$ isobar.

Characteristic effective two-nucleon processes are shown in Fig. 2. An instantaneous two-nucleon reference potential incorporates them, in an implicit average way, in its intermediate-range attraction, often modeled as sigma $(\sigma)$ exchange by one-boson exchange potentials. Thus, our previous strategy for achieving approximate phase equivalence with an underlying nucleonic reference potential amounted to taking these processes out from the intermediate-range attraction in an energy-independent way: The processes that the explicit channel coupling provides in an energydependent way are subtracted at a physically important reference energy, for which we chose zero kinetic energy; at that energy, phase equivalence is assured by construction. However, there is phase inequivalence at higher energies, and the phase inequivalence renders the potential not a realistic one. Changing the reference energy to higher values does not help much. Furthermore, we notice a dependence of calculated three-nucleon scattering observables on the choice of that reference energy. We therefore feel forced to create a new, this time well-fitted, coupled-channel potential, corresponding to a given nucleonic reference potential. This section describes the chosen procedure and the resulting interaction.

The underlying purely nucleonic reference potential is the charge-dependent CD-Bonn potential; it includes pion $(\pi)$,

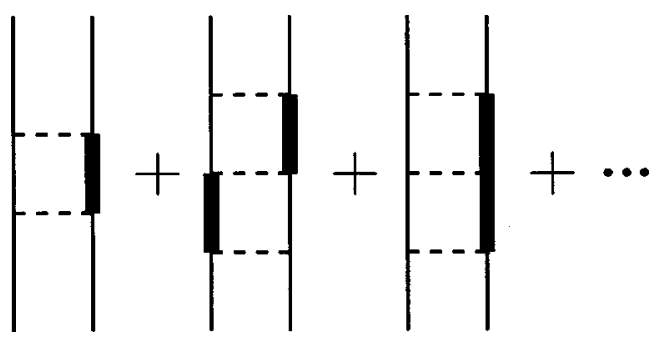

FIG. 2. Contributions to the effective two-nucleon interaction. The processes up to third order in the potential are shown. rho $(\rho)$ and omega $(\omega)$ mesons and, in addition, two effective scalar isoscalar $\sigma$ mesons, denoted by $\sigma_{1}$ and $\sigma_{2}$; it assumes vanishing coupling of the eta $(\eta)$ meson to the nucleon. The potential form, meson parameters, and regularizing hadronic form factors of CD Bonn are given in Ref. [9]. We choose the isospin triplet components of its coupledchannel extension in close correspondence to CD Bonn. The nucleonic part is taken over in form.

Furthermore, the transition potential of Fig. 1(b) from two-nucleon to nucleon- $\Delta$ states is based on $\pi$ and $\rho$ exchange and is taken to have the contributions

$$
\begin{aligned}
&\left\langle\mathbf{p}^{\prime}\right| v(N N \rightarrow N \Delta, \pi)|\mathbf{p}\rangle \\
&=- \frac{f_{\pi N N} f_{\pi N \Delta}}{(2 \pi)^{3} m_{\pi}^{2}} \boldsymbol{\tau}_{1} \cdot \mathbf{T}_{2} \frac{m_{N}}{\left(E^{\prime} E\right)^{1 / 2}} \frac{\boldsymbol{\sigma}_{1} \cdot \mathbf{p}_{E} \mathbf{S}_{2} \cdot \mathbf{p}_{E}}{\left(\mathbf{p}^{\prime}-\mathbf{p}\right)^{2}+m_{\pi}^{2}} \\
& \times \mathcal{F}_{\pi N}\left(\mathbf{p}^{\prime}, \mathbf{p}\right) \mathcal{F}_{\pi \Delta}\left(\mathbf{p}^{\prime}, \mathbf{p}\right), \\
&\left\langle\mathbf{p}^{\prime}\right| v(N N \rightarrow N \Delta, \rho)|\mathbf{p}\rangle \\
&=- \frac{f_{\rho N N} f_{\rho N \Delta}}{(2 \pi)^{3} m_{\rho}^{2}} \boldsymbol{\tau}_{1} \cdot \mathbf{T}_{2} \frac{m_{N}}{\left(E^{\prime} E\right)^{1 / 2}} \frac{\left(\boldsymbol{\sigma}_{1} \times \mathbf{p}_{E} \cdot\left(\mathbf{S}_{2} \times \mathbf{p}_{E}\right)\right.}{\left(\mathbf{p}^{\prime}-\mathbf{p}\right)^{2}+m_{\rho}^{2}} \\
& \quad \times \mathcal{F}_{\rho N}\left(\mathbf{p}^{\prime}, \mathbf{p}\right) \mathcal{F}_{\rho \Delta}\left(\mathbf{p}^{\prime}, \mathbf{p}\right)
\end{aligned}
$$

with the initial and final relative momenta $\mathbf{p}$ and $\mathbf{p}^{\prime}$,

$$
\begin{gathered}
E=\left(m_{N}^{2}+\mathbf{p}^{2}\right)^{1 / 2}, \\
E^{\prime}=\left(m_{N}^{2}+\mathbf{p}^{\prime 2}\right)^{1 / 2} \\
\mathbf{p}_{E}=\left[\left(E^{\prime}+m_{N}\right)\left(E+m_{N}\right)\right]^{1 / 2}\left(\frac{\mathbf{p}^{\prime}}{E^{\prime}+m_{N}}-\frac{\mathbf{p}}{E+m_{N}}\right)
\end{gathered}
$$

and the hadronic form factors

$$
\mathcal{F}_{\alpha B}\left(\mathbf{p}^{\prime}, \mathbf{p}\right)=\frac{\Lambda_{\alpha B}^{2}-m_{\alpha}^{2}}{\Lambda_{\alpha B}^{2}+\left(\mathbf{p}^{\prime}-\mathbf{p}\right)^{2}},
$$

$\boldsymbol{\sigma}(\boldsymbol{\tau})$ and $\mathbf{S}(\mathbf{T})$ being the nucleonic spin (isospin) operator and transition spin (isospin) operator from nucleonic to $\Delta$-isobar states with the reduced matrix elements $\left(\frac{1}{2}\|\boldsymbol{\sigma}\| \frac{1}{2}\right)$ $=\left(\frac{1}{2}\|\boldsymbol{\tau}\| \frac{1}{2}\right)=\sqrt{6}$ and $\left(\frac{3}{2}\|\mathbf{S}\| \frac{1}{2}\right)=\left(\frac{3}{2} \| \mathbf{T}|| \frac{1}{2}\right)=2$. In Eqs. (1) the second nucleon is turned into a $\Delta$ isobar; of course, the symmetrized transition contribution is added. The form factor (2d) acts at purely nucleonic vertices and at vertices with at least one $\Delta$ isobar; the subscript $B$ being $N$ or $\Delta$ distinguishes the two cases.

The exchange nucleon- $\Delta$ potential of Fig. 1(c) is based on $\pi$ and $\rho$ exchange and is taken to have the contributions 


$$
\begin{aligned}
&\left\langle\mathbf{p}^{\prime}\right| v(\Delta N \rightarrow N \Delta, \pi)|\mathbf{p}\rangle \\
&=- \frac{f_{\pi N \Delta}^{2}}{(2 \pi)^{3} m_{\pi}^{2}} \mathbf{T}_{1}^{\dagger} \cdot \mathbf{T}_{2} \frac{m_{N}}{\left(E^{\prime} E\right)^{1 / 2}} \frac{\mathbf{S}_{1}^{\dagger} \cdot \mathbf{p}_{E} \mathbf{S}_{2} \cdot \mathbf{p}_{E}}{\left(\mathbf{p}^{\prime}-\mathbf{p}\right)^{2}+m_{\pi}^{2}} \\
& \quad \times \mathcal{F}_{\pi \Delta}^{2}\left(\mathbf{p}^{\prime}, \mathbf{p}\right), \\
&\left\langle\mathbf{p}^{\prime}|v(\Delta N \rightarrow N \Delta, \rho)| \mathbf{p}\right\rangle \\
&=-\frac{f_{\rho N \Delta}^{2}}{(2 \pi)^{3} m_{\rho}^{2}} \mathbf{T}_{1}^{\dagger} \cdot \mathbf{T}_{2} \frac{m_{N}}{\left(E^{\prime} E\right)^{1 / 2}} \frac{\left(\mathbf{S}_{1}^{\dagger} \times \mathbf{p}_{E}\right) \cdot\left(\mathbf{S}_{2} \times \mathbf{p}_{E}\right)}{\left(\mathbf{p}^{\prime}-\mathbf{p}\right)^{2}+m_{\rho}^{2}} \\
& \quad \times \mathcal{F}_{\rho \Delta}^{2}\left(\mathbf{p}^{\prime}, \mathbf{p}\right) .
\end{aligned}
$$

The direct nucleon- $\Delta$ potential of Fig. 1(d) is based on $\pi, \rho$, $\omega$, and one $\sigma$ exchange and is taken to have the contributions

$$
\begin{aligned}
& \left\langle\mathbf{p}^{\prime}|v(N \Delta \rightarrow N \Delta, \pi)| \mathbf{p}\right\rangle \\
& =-\frac{f_{\pi N N} f_{\pi \Delta \Delta}}{(2 \pi)^{3} m_{\pi}^{2}} \boldsymbol{\tau}_{1} \cdot \boldsymbol{\tau}_{\Delta 2} \frac{m_{N}}{\left(E^{\prime} E\right)^{1 / 2}} \frac{\boldsymbol{\sigma}_{1} \cdot \mathbf{p}_{E} \boldsymbol{\sigma}_{\Delta 2} \cdot \mathbf{p}_{E}}{\left(\mathbf{p}^{\prime}-\mathbf{p}\right)^{2}+m_{\pi}^{2}} \\
& \times \mathcal{F}_{\pi N}\left(\mathbf{p}^{\prime}, \mathbf{p}\right) \mathcal{F}_{\pi \Delta}\left(\mathbf{p}^{\prime}, \mathbf{p}\right), \\
& \left\langle\mathbf{p}^{\prime}|v(N \Delta \rightarrow N \Delta, \rho)| \mathbf{p}\right\rangle \\
& =-\frac{f_{\rho N N} f_{\rho \Delta \Delta}}{(2 \pi)^{3} m_{\rho}^{2}} \boldsymbol{\tau}_{1} \cdot \boldsymbol{\tau}_{\Delta 2} \frac{m_{N}}{\left(E^{\prime} E\right)^{1 / 2}} \frac{\left(\boldsymbol{\sigma}_{1} \times \mathbf{p}_{E}\right) \cdot\left(\boldsymbol{\sigma}_{\Delta 2} \times \mathbf{p}_{E}\right)}{\left(\mathbf{p}^{\prime}-\mathbf{p}\right)^{2}+m_{\rho}^{2}} \\
& \times \mathcal{F}_{\rho N}\left(\mathbf{p}^{\prime}, \mathbf{p}\right) \mathcal{F}_{\rho \Delta}\left(\mathbf{p}^{\prime}, \mathbf{p}\right) \\
& +\frac{g_{\rho N N} g_{\rho \Delta \Delta}}{(2 \pi)^{3}} \boldsymbol{\tau}_{1} \cdot \boldsymbol{\tau}_{\Delta 2} \frac{\mathcal{F}_{\rho N}\left(\mathbf{p}^{\prime}, \mathbf{p}\right) \mathcal{F}_{\rho \Delta}\left(\mathbf{p}^{\prime}, \mathbf{p}\right)}{\left(\mathbf{p}^{\prime}-\mathbf{p}\right)^{2}+m_{\rho}^{2}}, \\
& \left\langle\mathbf{p}^{\prime}|v(N \Delta \rightarrow N \Delta, \sigma)| \mathbf{p}\right\rangle \\
& =-\frac{g_{\sigma N N} g_{\sigma \Delta \Delta}}{(2 \pi)^{3}} \frac{\mathcal{F}_{\sigma N}\left(\mathbf{p}^{\prime}, \mathbf{p}\right) \mathcal{F}_{\sigma \Delta}\left(\mathbf{p}^{\prime}, \mathbf{p}\right)}{\left(\mathbf{p}^{\prime}-\mathbf{p}\right)^{2}+m_{\sigma}^{2}}, \\
& \left\langle\mathbf{p}^{\prime}|v(N \Delta \rightarrow N \Delta, \omega)| \mathbf{p}\right\rangle \\
& =-\frac{g_{\omega N N} g_{\omega \Delta \Delta}}{(2 \pi)^{3}\left(2 m_{N}\right)^{2}} \frac{m_{N}}{\left(E^{\prime} E\right)^{1 / 2}} \frac{\left(\boldsymbol{\sigma}_{1} \times \mathbf{p}_{E}\right) \cdot\left(\boldsymbol{\sigma}_{\Delta 2} \times \mathbf{p}_{E}\right)}{\left(\mathbf{p}^{\prime}-\mathbf{p}\right)^{2}+m_{\omega}^{2}} \\
& \times \mathcal{F}_{\omega N}\left(\mathbf{p}^{\prime}, \mathbf{p}\right) \mathcal{F}_{\omega \Delta}\left(\mathbf{p}^{\prime}, \mathbf{p}\right) \\
& +\frac{g_{\omega N N} g_{\omega \Delta \Delta}}{(2 \pi)^{3}} \frac{\mathcal{F}_{\omega N}\left(\mathbf{p}^{\prime}, \mathbf{p}\right) \mathcal{F}_{\omega \Delta}\left(\mathbf{p}^{\prime}, \mathbf{p}\right)}{\left(\mathbf{p}^{\prime}-\mathbf{p}\right)^{2}+m_{\omega}^{2}},
\end{aligned}
$$

$\boldsymbol{\sigma}_{\Delta}\left(\boldsymbol{\tau}_{\Delta}\right)$ being the $\Delta$-isobar spin (isospin) operator with the reduced matrix element $\left(\frac{3}{2}\left\|\boldsymbol{\sigma}_{\Delta}\right\| \frac{3}{2}\right)=\left(\frac{3}{2}\left\|\tau_{\Delta}\right\| \frac{3}{2}\right)=2 \sqrt{15}$.

The potential forms relating to the $\Delta$ isobar differ from those used by us previously in several respects.

(1) We do not derive forms (1), (3), and (4) cleanly from field theory, but postulate them by substituting spin and isospin operators in the nucleonic ones of CD Bonn with the corresponding transition and diagonal $\Delta$-isobar operators.
They are made to carry the same relativistic phase-space factors as CD Bonn; for simplicity no distinction between nucleon and $\Delta$-isobar masses is made in these factors. Their nonlocal relativistic forms are especially important for the tensor force; their local approximations change them drastically off shell: Locality makes the tensor-force part substantially stronger off shell [9].

(2) The regularizing hadronic form factors are dipole ones for each meson exchange as in CD Bonn.

(3) The direct hadronic nucleon- $\Delta$ potential (4) is taken to be nonzero, in contrast to our assumptions in Refs. [1-6]. Furthermore, a point Coulomb contribution is added, appropriate for the charge content of the channel.

The fit of the isospin triplet part of the two-baryon coupled-channel potential proceeds as follows and thereby yields the following characteristics for the resulting potential.

(1) The CD-Bonn potential form is adopted as nucleonic part. The parameters of the $\sigma_{1}$ and $\sigma_{2}$ exchanges are retuned. However, in the ${ }^{3} P_{0}$ and ${ }^{3} P_{1}$ partial waves the readjustment of the $\sigma_{1}$ and $\sigma_{2}$ parameters alone is not enough; there, also the $\omega$ parameters have to be retuned slightly. Thus, the readjustment of the $\sigma_{1}, \sigma_{2}$ and $\omega$ exchanges is partial-wave dependent. The resulting parameters are given in Table III of the Appendix. The $\sigma_{1}$ and $\sigma_{2}$, but not the $\omega$ parameters of the purely nucleonic CD Bonn are also partial-wave dependent.

(2) In the potential parts referring to the $\Delta$ isobar, the parameters, except for the $\sigma$ coupling strength, are chosen according to empirical values or quark counting rules; they are summarized in Table IV of the Appendix. Since these parameters are not subjected to the fit, the fit is not allowed to return to the purely nucleonic reference potential, i.e., to choose these parameters to vanish. The coupling strength of $\sigma$, in fact, the combination $g_{\sigma N N} g_{\sigma \Delta \Delta} / 4 \pi$, is the proper fit parameter; it is allowed to be partial-wave dependent as in CD Bonn; the resulting parameters of $\sigma$ exchange are given in Table $\mathrm{V}$ of the Appendix. The coupled-channel potential is charge dependent. However, the potential contributions related to the $\Delta$ isobar are chosen as charge independent; the charge dependence of the complete coupled-channel interaction results from the purely nucleonic part.

(3) The CD-Bonn potential can be considered a very reliable energy-dependent phase-shift analysis of nucleonnucleon scattering data below $350 \mathrm{MeV}$, known up to the year 2000. It can be considered an update of the corresponding Nijmegen phase shift analysis which is based on data up to 1993 . The coupled-channel potential of this paper is therefore tuned to the phase shifts of CD Bonn; however, the resulting $\chi^{2}$ values are calculated with respect to the proper data as in Ref. [9]. Furthermore, the actual fit sequence for the different charge states is the same as in Ref. [9].

(4) The point Coulomb interaction is added in the partial waves with two charged baryons, i.e., in the coupled protonproton $(p p)$ and $p \Delta^{+}$partial waves and in the $p \Delta^{-}$partial waves coupled to the neutron-neutron $(n n)$ partial waves. The long range Coulomb potential is cut off at a radius $R$, outside the range of the hadronic potentials. In the $p p$ partial waves, the proper Coulomb boundary conditions are exactly 
TABLE I. $\chi^{2}$ values for all potentials used in this paper. The coupled-channel potential CD Bonn $+\Delta$ is the fitted one. The other coupled-channel potentials are constructed without fit.

\begin{tabular}{lccc}
\hline \hline & $\chi^{2} /$ datum $(p p)$ & $\chi^{2} /$ datum $(n p)$ & $\chi^{2} /$ datum $(p p+n p)$ \\
\hline CD Bonn & 1.01 & 1.02 & 1.02 \\
CD Bonn $+\Delta$ & 1.01 & 1.02 & 1.02 \\
CD Bonn $+\Delta$ (sub1) & 10.5 & 2.36 & 6.34 \\
CD Bonn $+\Delta$ (sub2) & 23.8 & 4.11 & 13.8 \\
\hline \hline
\end{tabular}

restored from the cutoff ones; with respect to the cutoff in $N \Delta$ partial waves, the independence of results from that cutoff is numerically established.

(5) The fit aims first at the $p p$ potential with channel coupling, since the $p p$ data are the most accurate ones. The $p p$ potential is fitted to the CD-Bonn $p p$ phase shifts, using the Nijmegen $p p$ error matrix [13] for determining an intermediate $\chi^{2}$. The subsequent direct comparison with all experimental $p p$ data below $350 \mathrm{MeV}$, available in the year 2000 , yields $\chi^{2} /$ datum $=1.01$, very close to that of the original CD Bonn. Thus, there is no need for any further tuning of parameters in comparison with the proper $p p$ data.

(6) The coupled-channel potential is charge dependent as CD Bonn. Its parameters in isospin triplet partial waves with isospin projection $M_{T}=1$, fitted in step 5, are transcribed to the neutron-proton $(n p)$, i.e., $M_{T}=0$, and the $n n$, i.e., $M_{T}$ $=-1$, parts in the same way as for CD Bonn, i.e., omitting Coulomb, except in the $p \Delta^{-}$channel coupled to $n n$, correspondingly replacing the masses of the nucleons and adjusting the coupling constants of the $\sigma_{1}$ and $\sigma_{2}$ bosons such that the phase shift differences, predicted by the chargeindependence and the charge-symmetry breaking of $\mathrm{CD}$ Bonn, are reproduced. The subsequent direct comparison with experimental $n p$ data below $350 \mathrm{MeV}$, available in the year 2000 , yields $\chi^{2} /$ datum $=1.02$, again very close to that of the original CD Bonn. Thus, there is no need for any further tuning of parameters in comparison with the proper $n p$ data. Furthermore, the resulting ${ }^{1} S_{0} n n$ scattering length, i.e., $-18.95 \mathrm{fm}$, agrees well with the experimental one of Ref. [14], i.e., with $-18.9 \pm 0.4 \mathrm{fm}$, within the experimental error bars.

The meson parameters resulting from the fit are collected in the tables of the Appendix. The overall fit yields a $\chi^{2} /$ datum $=1.02$. Thus, the coupled-channel potential of this paper is as realistic as any of the modern nucleonic potentials; it is phase equivalent with CD Bonn as nucleonic reference potential in the limits of the fit. Nevertheless, a word of caution is appropriate: The fit is based on nucleon-nucleon scattering data below pion-production threshold, whereas the nucleon- $\Delta$ channel is the remainder of the description of inelasticity yielding single-pion production. Thus, those physics data for which the nucleon- $\Delta$ channel is most important are not used yet for determining its properties. The coupled-channel potential of this paper is applicable only for phenomena below pion-production threshold in the same way as the nucleonic reference potential CD Bonn; both are unrealistic beyond pion-production threshold. Of course, this fact is unfortunate, but its repair is far beyond the limited scope of this paper; only for reasons of curiosity, the Appendix also discusses characteristic predictions of the coupledchannel potential for the energy domain, where single-pion inelasticity is important.

TABLE II. Hadronic properties of ${ }^{3} \mathrm{H}$ (top) and ${ }^{3} \mathrm{He}$ (bottom). The $\Delta$-isobar effect on the binding energy $E_{B}$ is split into the two-nucleon dispersion $\Delta E_{2}$ and the effective three-nucleon force effect $\Delta E_{3}$. The probability $P_{\mathcal{L}}, \mathcal{L}$ being $S, S^{\prime}, P$ or $D$, refers to purely nucleonic $\mathcal{T}=\frac{1}{2}$ wave function components with definite total three-nucleon orbital angular momentum and definite permutation symmetry according to Refs. [16,17], $P_{3 / 2}$ to the $\mathcal{T}=\frac{3}{2}$ wave function component arising from charge dependence, and $P_{\Delta}$ to the wave function components with $\Delta$-isobar configurations. All energies are given in $\mathrm{MeV}$, all probabilities are given in percent; always three digits are quoted, only for the very small quantity $P_{3 / 2}$ four digits are quoted; they appear converged.

\begin{tabular}{|c|c|c|c|c|c|c|c|c|c|c|}
\hline & & $E_{B}$ & $\Delta E_{2}$ & $\Delta E_{3}$ & $P_{S}$ & $P_{S^{\prime}}$ & $P_{P}$ & $P_{D}$ & $P_{3 / 2}$ & $P_{\Delta}$ \\
\hline \multirow[t]{5}{*}{${ }^{3} \mathrm{H}:$} & CD Bonn & -8.004 & & & 91.621 & 1.307 & 0.047 & 7.020 & 0.0048 & \\
\hline & CD Bonn $+\Delta$ & -8.297 & 0.513 & -0.806 & 89.922 & 1.301 & 0.064 & 7.216 & 0.0045 & 1.493 \\
\hline & CD Bonn $+\Delta($ sub1 $)$ & -8.515 & 0.353 & -0.864 & 89.911 & 1.173 & 0.067 & 7.293 & 0.0047 & 1.552 \\
\hline & CD Bonn $+\Delta($ sub2) & -8.271 & 0.648 & -0.915 & 88.799 & 1.207 & 0.073 & 7.237 & 0.0045 & 2.680 \\
\hline & Experiment & -8.482 & & & & & & & & \\
\hline \multirow[t]{5}{*}{${ }^{3} \mathrm{He}:$} & CD Bonn & -7.258 & & & 91.403 & 1.538 & 0.046 & 7.002 & 0.0111 & \\
\hline & CD Bonn $+\Delta$ & -7.541 & 0.483 & -0.766 & 89.776 & 1.515 & 0.063 & 7.197 & 0.0104 & 1.439 \\
\hline & CD Bonn $+\Delta($ sub1 $)$ & -7.752 & 0.328 & -0.822 & 89.781 & 1.370 & 0.066 & 7.274 & 0.0105 & 1.499 \\
\hline & CD Bonn $+\Delta($ sub2) & -7.521 & 0.601 & -0.864 & 88.711 & 1.412 & 0.072 & 7.216 & 0.0102 & 2.579 \\
\hline & Experiment & -7.718 & & & & & & & & \\
\hline
\end{tabular}




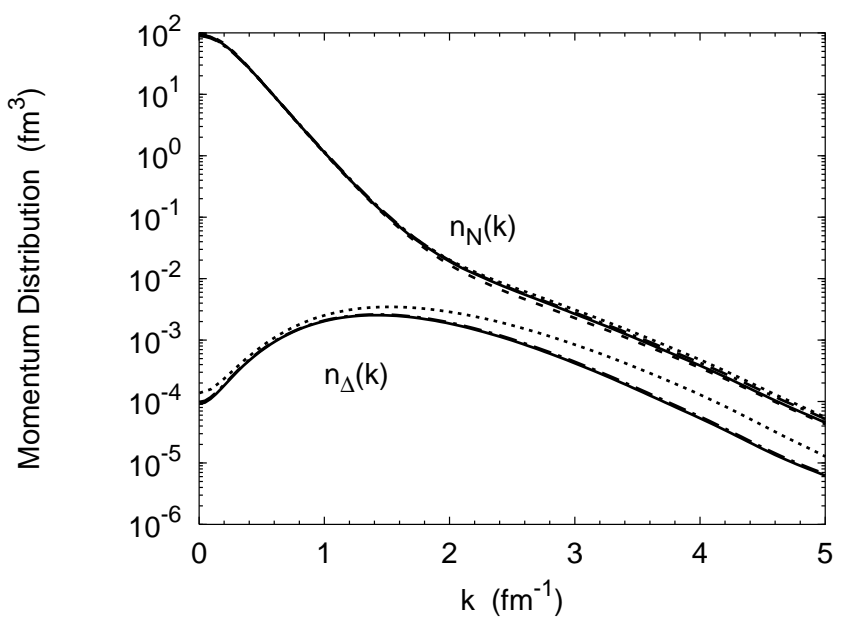

FIG. 3. ${ }^{3} \mathrm{H}$ nucleon and $\Delta$-isobar momentum distributions $n_{N}(k)$ and $n_{\Delta}(k)$ as functions of the magnitude of the singleparticle momentum $k$. The distributions are normalized such that $\int_{0}^{\infty} d k k^{2}\left[n_{N}(k)+n_{\Delta}(k)\right]=3$. Compared are predictions of the different coupled-channel potentials, i.e., CD Bonn $+\Delta$ (solid curves), CD Bonn $+\Delta$ (sub1) (dashed-dotted curves), CD Bonn $+\Delta$ (sub2) (dotted curves). The solid and dashed-dotted curves are indistinguishable in the plot. Results for $n_{N}(k)$ based on the nucleonic CD Bonn potential without $\Delta$-isobar excitation are given by the dashed curve.
In the following we present results for the three-nucleon bound state and for nucleon-deuteron scattering derived from the new realistic coupled-channel potential with $\Delta$-isobar excitation, denoted in the following as CD Bonn $+\Delta$. We shall give three additional results for comparison. We shall give results for the nucleonic reference potential CD Bonn in order to isolate $\Delta$-isobar effects. We shall also give results for coupled-channel potentials constructed according to our old subtraction techniques $[1,15]$ without fit; both are phaseequivalent at zero kinetic energy only. One version, denoted in the following as CD Bonn $+\Delta$ (sub1), is based on the contributions (1)-(4) with the parameters of the Appendix; its $\chi^{2} /$ datum $=6.34$ is poor compared to the new coupledchannel potential. The other version is that employed in Ref. [5]; it will be denoted as CD Bonn $+\Delta$ (sub2); it is based on a local transition potential without diagonal nucleon- $\Delta$ contributions; its $\chi^{2} /$ datum $=13.8$ is even poorer. The partial and the complete $\chi^{2}$ values of all coupled-channel potentials, used in this paper, and of the nucleonic reference potential CD Bonn are collected in Table I.

\section{HADRONIC PROPERTIES OF THREE-NUCLEON BOUND STATE}

The calculation of the three-nucleon bound state follows our technique of Ref. [5]. The dynamic input is charge de-

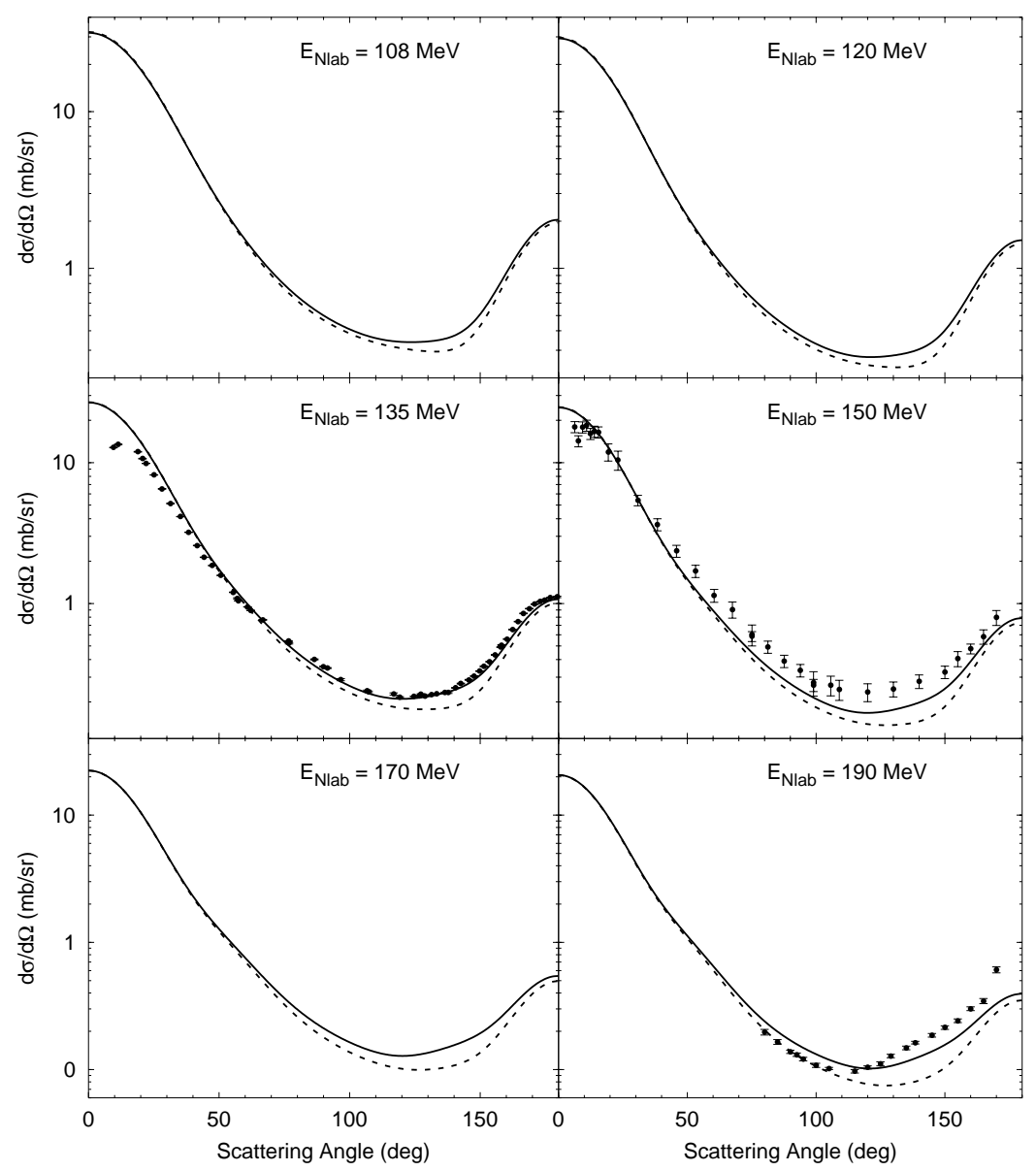

FIG. 4. Differential cross section of elastic nucleon-deuteron scattering at 108, 120, 135, 150, 170, and $190 \mathrm{MeV}$ nucleon lab energy as function of the c.m. scattering angle. Results of the coupled-channel potential with $\Delta$-isobar excitation (solid curves) are compared with results of the CD-Bonn potential (dashed curves). The experimental data are from Refs. [18-20] and refer to proton-deuteron scattering at 135,146 , and $198 \mathrm{MeV}$ nucleon lab energy, respectively. 


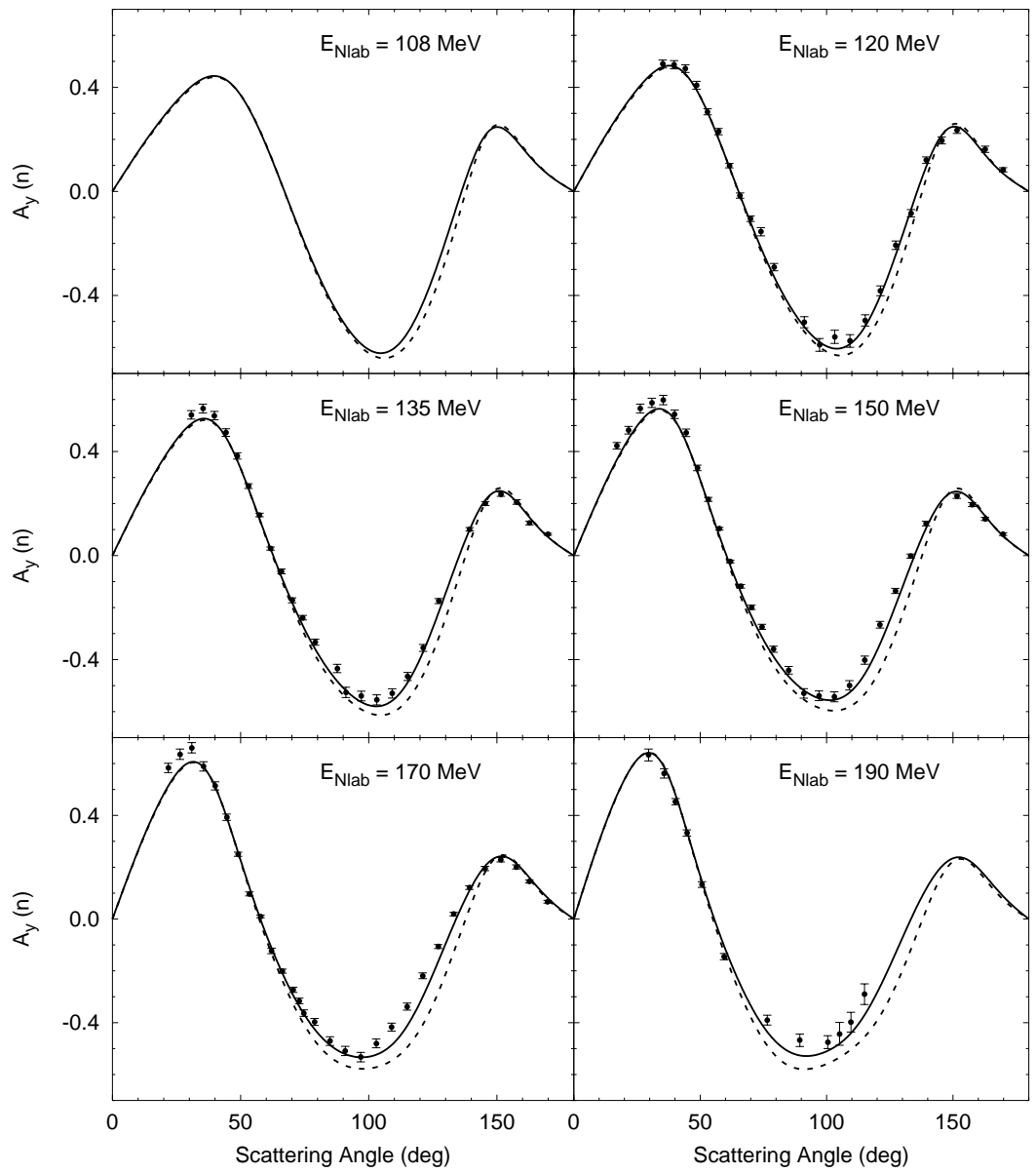

FIG. 5. Nucleon analyzing power $A_{y}(n)$ of elastic nucleon-deuteron scattering at 108, 120, $135,150,170$, and $190 \mathrm{MeV}$ nucleon lab energy as a function of the c.m. scattering angle. Results of the coupled-channel potential with $\Delta$-isobar excitation (solid curves) are compared with results of the CD-Bonn potential (dashed curves). The experimental data are from Refs. $[22,23]$ and refer to proton-deuteron scattering. pendent; thus, the calculation is done separately for ${ }^{3} \mathrm{He}$ and ${ }^{3} \mathrm{H}$. Furthermore, we include the point Coulomb interaction by cutting off its technically dangerous long tail and adding that cutoff Coulomb to the hadronic potential; the independence of the results from the cutoff radius $R$ for sufficiently large values is established; we find $R=15 \mathrm{fm}$ large enough. The point Coulomb interaction has to be included not only between the two protons in ${ }^{3} \mathrm{He}$ but also in channels with a $\Delta$ isobar, i.e., in the $n p \Delta^{+}$channel of ${ }^{3} \mathrm{He}$ and in the $p p \Delta^{-}$ channel of ${ }^{3} \mathrm{H}$. Partial waves up to total two-baryon angular momentum $I=6$ in purely nucleonic channels and up to $I$ $=4$ in nucleon- $\Delta$ channels are taken into account. The charge dependence of the nucleon-nucleon and the Coulomb interactions is treated exactly in partial waves up to $I=2$, yielding total isospin $\mathcal{T}=\frac{3}{2}$ channels; in other two-baryon isospin triplet partial waves the charge dependence is treated approximately, i.e., without coupling to $\mathcal{T}=\frac{3}{2}$ states; in those higher partial waves the coupling to $\mathcal{T}=\frac{3}{2}$ states was checked to be quantitatively irrelevant. The results appear fully converged with all those truncations on partial waves.

Results on binding-energy contributions are collected in Table II. We notice, as observed and discussed already long ago [1], two sizable $\Delta$-isobar effects on binding which partially cancel each other, i.e., the repulsive dispersion $\Delta E_{2}$ and the attractive three-nucleon force effect $\Delta E_{3}$ proper. Though the purely nucleonic reference potential CD Bonn misses the three-nucleon binding by rather little and the
$\Delta$-isobar effects arising from the new coupled-channel potential are beneficial, still, they are unable to account for the missing binding in full. The experimental ${ }^{3} \mathrm{He}-{ }^{3} \mathrm{H}$ bindingenergy difference is $0.764 \mathrm{MeV}$; most of it is due to the Coulomb interaction in ${ }^{3} \mathrm{He}$. When calculating ${ }^{3} \mathrm{H}$ with a charge symmetric hadronic interaction, i.e., with the same hadronic part for $p p$ and $n n$, Coulomb alone yields a binding-energy difference of $0.685 \mathrm{MeV}$. The charge asymmetry of the coupled-channel potential makes an additional contribution of $0.059 \mathrm{MeV}$ and the kinematic effects due to proton and neutron mass difference add additional 0.012 $\mathrm{MeV}$, yielding a total binding-energy difference of 0.756 $\mathrm{MeV}$. The theoretical value agrees quite well with the experimental value of $0.764 \mathrm{MeV}$.

The two constructions of coupled-channel potentials without fit, CD Bonn $+\Delta$ (sub1) and CD Bonn $+\Delta$ (sub2), are unable to account for the three-nucleon binding energy with $\Delta$-isobar effects accurate enough; both fail especially with respect to the two-nucleon dispersive repulsion $\Delta E_{2}, \mathrm{CD}$ Bonn $+\Delta$ (sub2) also with respect to $P_{\Delta}$ and to the momentum distribution of the $\Delta$ isobar as displayed in Fig. 3 for ${ }^{3} \mathrm{H}$, together with the nucleonic momentum distribution.

\section{IV. $\Delta$-ISOBAR EFFECTS IN ELASTIC NUCLEON- DEUTERON SCATTERING AND BREAKUP}

We present results for spin-averaged and spin-dependent observables of elastic nucleon-deuteron $(N d)$ scattering and 


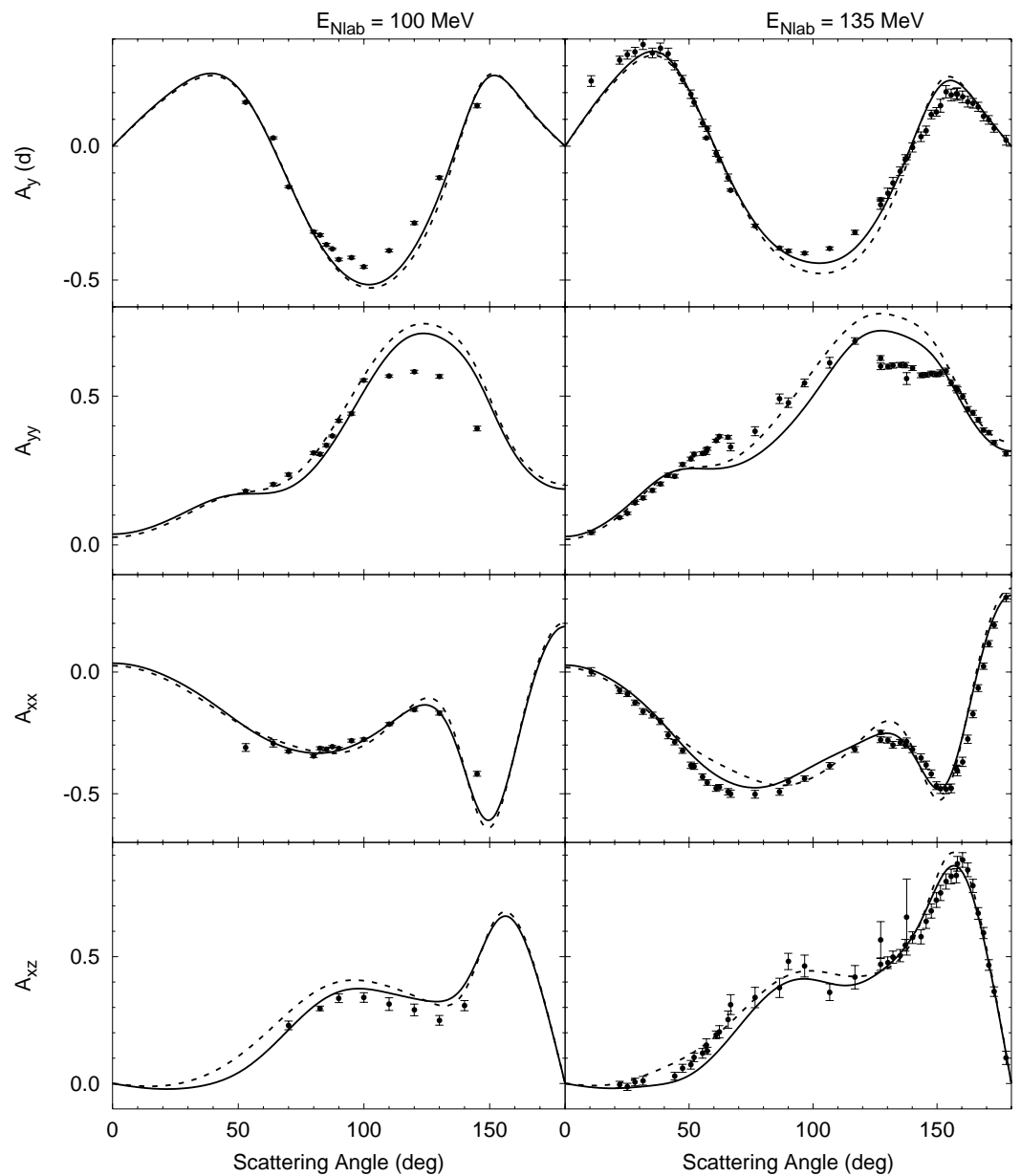

FIG. 6. Deuteron analyzing powers $A_{y}(d)$, $A_{y y}, A_{x x}$, and $A_{x z}$ of elastic nucleon-deuteron scattering at $100 \mathrm{MeV}$ (left side) and $135 \mathrm{MeV}$ (right side) nucleon lab energy as functions of the c.m. scattering angle. Results of the coupledchannel potential with $\Delta$-isobar excitation (solid curves) are compared with results of the CDBonn potential (dashed curves). The experimental data are from Ref. [18] and refer to protondeuteron scattering. breakup using the developed coupled-channel potential. The solution of the nucleon-deuteron scattering equations follows our technique of Ref. [5]. The shorthand for specifying the kinematics to which break-up observables refer is taken over from Ref. [4]. The calculations omit the Coulomb potential between charged baryons. The theoretical description is charge dependent. For $p d$ data the $p p$ and $n p$ parts of the interaction are used, for $n d$ data the $n n$ and $n p$ parts; except for very small energies, where the omission of Coulomb is fatal anyhow, both calculations yield results, indistinguishable in plots. The charge dependence of the nucleon-nucleon interaction is treated exactly in the ${ }^{1} S_{0}$ partial wave, yielding total isospin $\mathcal{T}=\frac{3}{2}$ channels; in the other two-baryon isospin triplet partial waves up to $I=4$ the charge dependence is treated approximately, i.e., without coupling to $\mathcal{T}=\frac{3}{2}$ states; this approximate treatment weights the components of the isospin triplet partial waves in the ratio $\frac{1}{3}: \frac{2}{3}$ for the $n p$ and the $p p$ (or $n n$ ) parts; in those higher partial waves the coupling to $\mathcal{T}=\frac{3}{2}$ states was checked to be quantitatively irrelevant. Partial waves up to total two-baryon angular momentum $I=5$ in purely nucleonic channels and up to $I=4$ in nucleon- $\Delta$ channels and up to total three-baryon angular momentum $\mathcal{J}=\frac{31}{2}$ are taken into account. The results appear fully converged with respect to higher two-baryon angular momenta $I$, with respect to higher three-baryon angular momenta $\mathcal{J}$ and with respect to $\Delta$-isobar coupling on the scale of accuracy which present-day experimental data require with only one exception: some break-up observables at 135 and $200 \mathrm{MeV}$ nucleon lab energy still show, in some kinematical regimes, a residual dependence on the cutoff in $I$ and $\mathcal{J}$; the results presented in figures for break-up observables at those higher energies are therefore based on the cutoffs $I$ $=6$ and $\mathcal{J}=\frac{51}{2}$. In the following, we focus on the $\Delta$-isobar effects in observables.

\section{A. Elastic nucleon-deuteron scattering}

We concentrate on observables between 100 and 190 $\mathrm{MeV}$ nucleon lab energy; this is an energy regime in which noticeable $\Delta$-isobar effects are expected, in contrast to lower energies. Figures 4 and 5 study the evolution of the $\Delta$-isobar effect on the spin-averaged differential cross section and on the nucleon analyzing power at 108, 120, 135, 150, 170, and $190 \mathrm{MeV}$ nucleon lab energy. There is a clear disagreement in the diffraction minima between experiment and theory based on the purely nucleonic CD-Bonn potential, the Sagara discrepancy; the inclusion of the $\Delta$ isobar reduces that discrepancy significantly, though it is unable to remove it in full for higher energies. In addition to the experimental data [1820] shown in Fig. 4 there exist also new, but still preliminary ones [21]; we expect them to confirm our conclusion on the repair of the Sagara discrepancy; at $135 \mathrm{MeV}$ nucleon lab energy they will lie higher than those of Ref. [18].

Figures 6 and 7 show deuteron analyzing powers, nucleon 

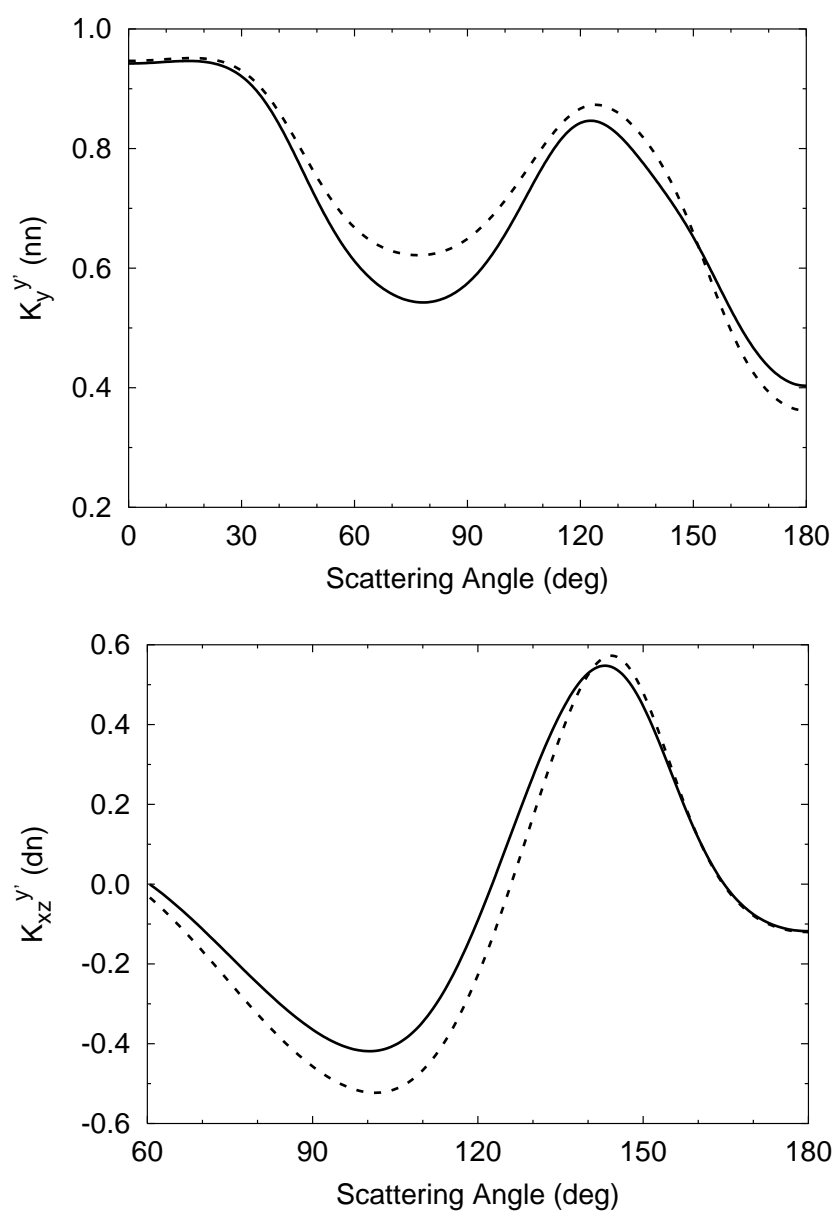

FIG. 7. Nucleon to nucleon and deuteron to nucleon polarization transfer coefficients $K_{y}^{y^{\prime}}(n n)$ and $K_{x z}^{y^{\prime}}(d n)$ of elastic nucleondeuteron scattering at $135 \mathrm{MeV}$ nucleon lab energy as functions of the c.m. scattering angle. Results of the coupled-channel potential with $\Delta$-isobar excitation (solid curves) are compared with results of the CD-Bonn potential (dashed curves).

to nucleon, and deuteron to nucleon polarization transfer coefficients.

Besides the quoted published experimental data, further experiments on the observables of Fig. 7 have been done, though they are not fully analyzed yet [24] or are being planned. The seen $\Delta$-isobar effects are not always beneficial.

Instead of letting the $\Delta$ isobar yield an effective threenucleon force besides other $\Delta$-isobar effects, Refs. [18,22] add an irreducible three-nucleon force to the purely nucleonic two-nucleon interaction; the two-pion exchange Tucson-Melbourne potential, i.e., TM' with revisited parameters, repairing a substantial violation of chiral symmetry, is the favorable choice. When comparing our results with the predictions of Refs. $[18,22]$ for the observables of Figs. 4-6, we observe an encouraging qualitative agreement. The agreement is even quantitative for the differential cross sections of Fig. 4. The only qualitative disagreement is found for the deuteron analyzing power $A_{x x}$ of Fig. 6, there the full $\Delta$-isobar effect and the effect of TM' go into opposite directions.

\section{B. Nucleon-deuteron breakup}

Very sizable effects, arising from the three-nucleon force and therefore giving information on it, are hoped to be seen in nucleon-deuteron breakup. This hope is a general expectation. Using the coupled-channel potential as our theoretical tool, we are not able to confirm that high expectation.

First, $\Delta$-isobar effects on the total break-up cross section are unspectacular; we do not present our results in figures.

Second, we show characteristic spin-averaged and spindependent observables for $65 \mathrm{MeV}$ nucleon lab energy in Fig. 8, for which data exist. $\Delta$-isobar effects are small; they appear to be most pronounced in collinearity configurations, as already pointed out in Refs. [4,5]. In contrast, there is almost no $\Delta$-isobar effects in star and quasi-free-scattering configurations. This is typical also for higher energies; we do not document our results on that finding.

Third, we do not repeat the impressive search for threenucleon force effects carried out in Ref. [26]. In Ref. [26] dramatic effects were seen in particular, experimentally not easily accessible kinematics regions, but they were based on a Tucson-Melbourne potential with questionable parameters; results obtained for CD Bonn together with the modified TM' three-nucleon force show much milder effects. Our own $\Delta$-isobar effects are pretty consistent with the latter ones. We give examples for our results with rather modest $\Delta$-isobar effects in Figs. 9 and 10.

\section{Separation of $\Delta$-isobar effects}

The two-nucleon dispersion due to the $\Delta$ isobar and the three-nucleon force mediated by the $\Delta$ isobar are usually competing mechanisms. We see that competition clearly in three-nucleon binding. We saw that competition already previously in three-nucleon scattering [27]; we confirm that competition in this paper for the new coupled-channel potential. Figure 11 give characteristic results; they are not obtained from perturbation theory as in Ref. [27], but from full results forming differences of results for observables.

\section{COMPARISON WITH PREVIOUS COUPLED-CHANNEL POTENTIALS}

At very low energies, i.e., below $10 \mathrm{MeV}$ nucleon lab energy, some scattering observables scale with three-nucleon binding and thereby show $\Delta$-isobar effects; we are not interested in those scaling effects which the $\Delta$ isobar yields due to the resulting additional binding. At low energies, i.e., up to about $50 \mathrm{MeV}$ nucleon lab energy, $\Delta$-isobar effects remain small; they are at least qualitatively the same for all coupledchannel potentials discussed in the past and in this paper. At higher energies $\Delta$-isobar effects become more visible and there the realistic nature of the employed coupled-channel potential becomes important. Figure 12 gives examples. We emphasize, we give worst cases, i.e., cases in which the spread of results is maximal. In the given examples, the coupled-channel potentials constructed according to the old subtraction scheme can overestimate the $\Delta$-isobar effect sizably. 

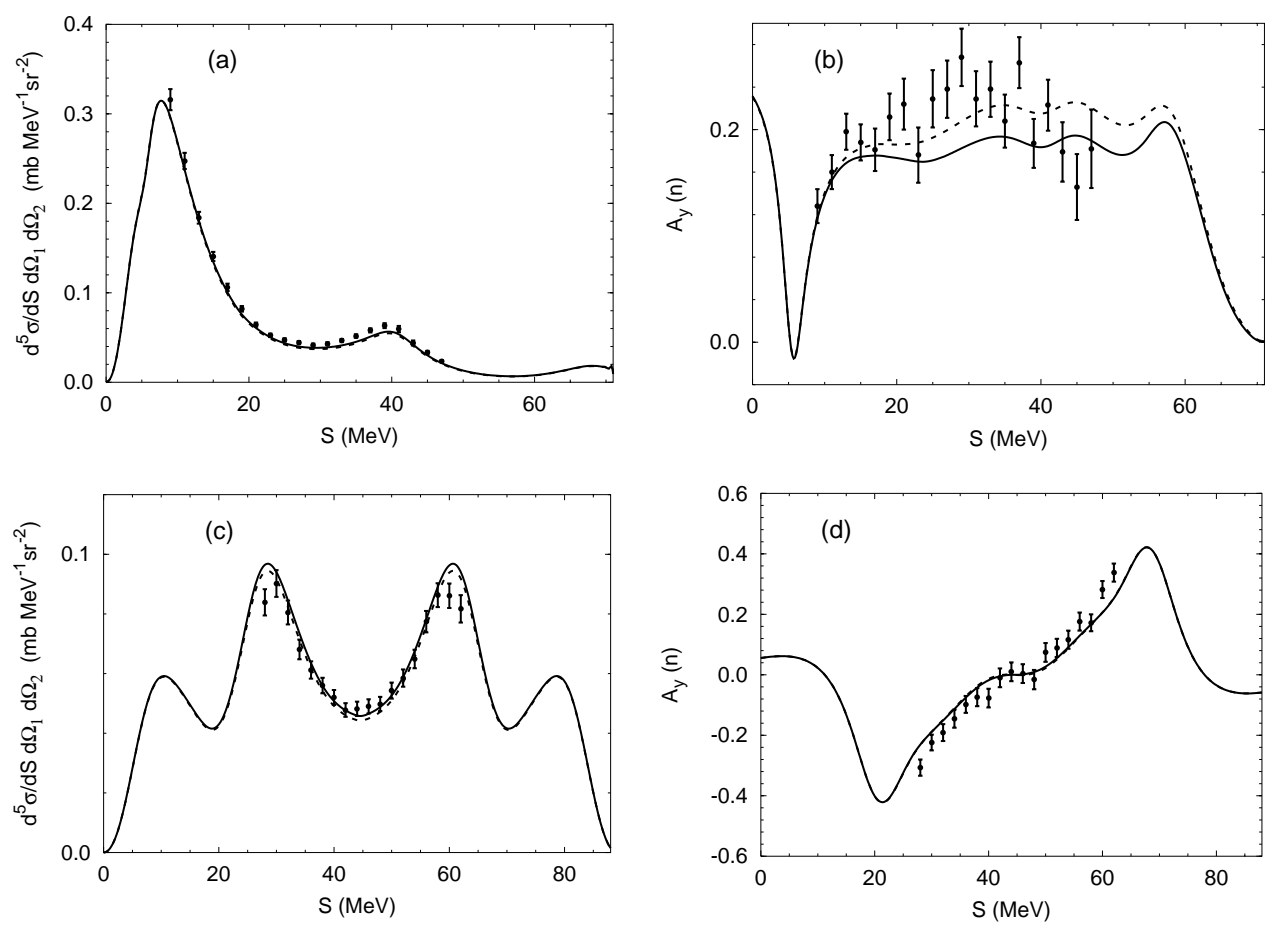

FIG. 8. Differential cross section and nucleon analyzing power $A_{y}(n)$ of nucleon-deuteron breakup at $65 \mathrm{MeV}$ nucleon lab energy as functions of the arclength $S$ along the kinematical curve for collinear configurations: (a,b) configuration $\left(30.0^{\circ}, 98.0^{\circ}, 180.0^{\circ}\right)$ and $(\mathrm{c}, \mathrm{d})$ configuration $\left(59.5^{\circ}, 59.5^{\circ}, 180.0^{\circ}\right)$. Results of the coupled-channel potential with $\Delta$-isobar excitation (solid curve) are compared with results of the CD-Bonn potential (dashed curve). The experimental data are from Ref. [25] and refer to proton-deuteron scattering.
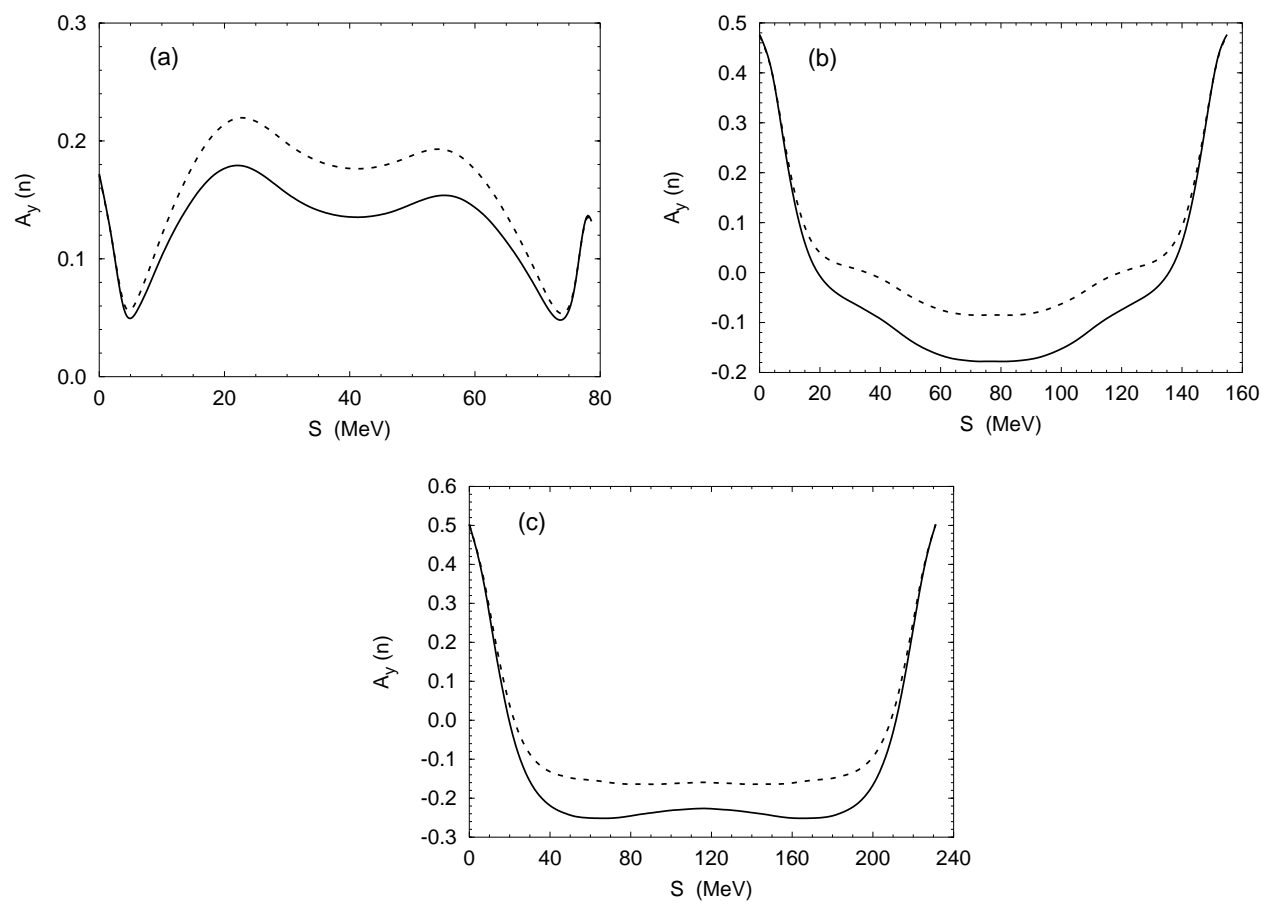

FIG. 9. Nucleon analyzing power $A_{y}(n)$ of nucleon-deuteron breakup as a function of the arclength $S$ along the kinematical curve for three different nucleon lab energies: (a) $65 \mathrm{MeV}$, configuration $\left(20^{\circ}, 20^{\circ}, 40^{\circ}\right)$; (b) $135 \mathrm{MeV}$, configuration $\left(25^{\circ}, 25^{\circ}, 0^{\circ}\right)$, and (c) $200 \mathrm{MeV}$, configuration $\left(25^{\circ}, 25^{\circ}, 0^{\circ}\right)$. Results of the coupled-channel potential with $\Delta$-isobar excitation (solid curve) are compared with results of the CD-Bonn potential (dashed curve). 

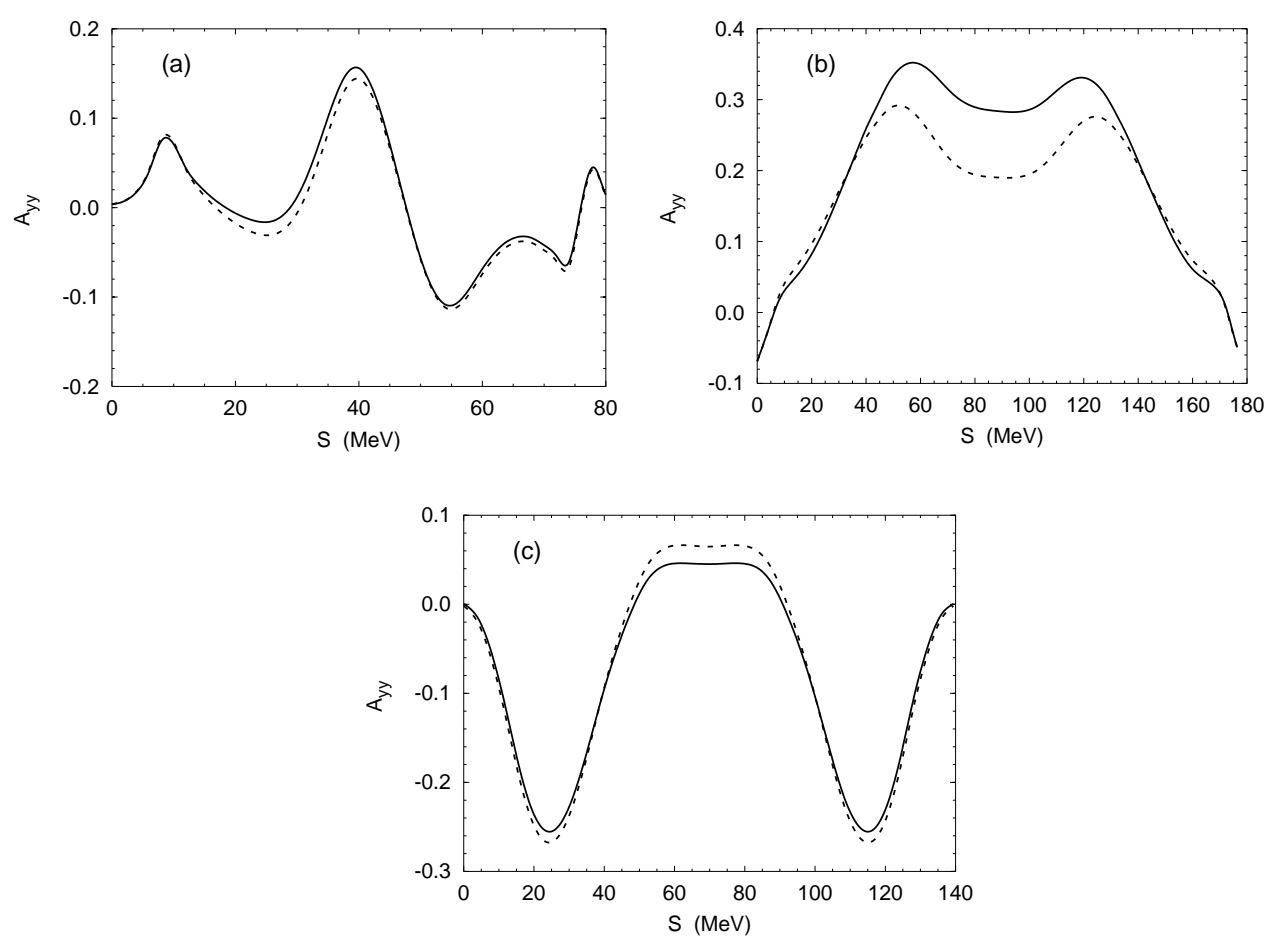

FIG. 10. Deuteron analyzing power $A_{y y}$ of nucleon-deuteron breakup as a function of the arclength $S$ along the kinematical curve for three different nucleon lab energies: (a) $65 \mathrm{MeV}$, configuration $\left(40^{\circ}, 30^{\circ}, 140^{\circ}\right)$; (b) $135 \mathrm{MeV}$, configuration $\left(15^{\circ}, 15^{\circ}, 20^{\circ}\right)$, and (c) $200 \mathrm{MeV}$, configuration $\left(45^{\circ}, 45^{\circ}, 0^{\circ}\right)$. Results of the coupled-channel potential with $\Delta$-isobar excitation (solid curve) are compared with results of the CD-Bonn potential (dashed curve).

\section{SUMMARY AND CONCLUSIONS}

This paper developed a new coupled-channel potential with single $\Delta$-isobar excitation. The nucleonic reference potential is the charge-dependent CD-Bonn potential; the coupled-channel potential is itself charge dependent in the nucleonic partial waves up to two-baryon total angular momentum $I=4$. All parts of the coupled-channel potential connected with the $\Delta$ isobar are based on meson exchange as CD Bonn.

The $\chi^{2} /$ datum resulting from the fit is with 1.02 as good as for the best present-day purely nucleonic potentials. The fit uses two-nucleon scattering data up to $350 \mathrm{MeV}$ nucleon lab energy, i.e., data up to pion-production threshold. The quality of the fit is gratifying, though low-energy elastic nucleon-nucleon scattering data do not form a convincing physics basis to constrain the properties of inelastic channels stringently. The coupled-channel potential and the nucleonic reference potential CD Bonn are both only applicable to phenomena below pion-production threshold.

Within the limits of the given $\chi^{2}$ the new coupled-channel potential and CD Bonn are phase equivalent. The developed new coupled-channel potential is a substantial advance, compared with our traditional construction of coupled-channel
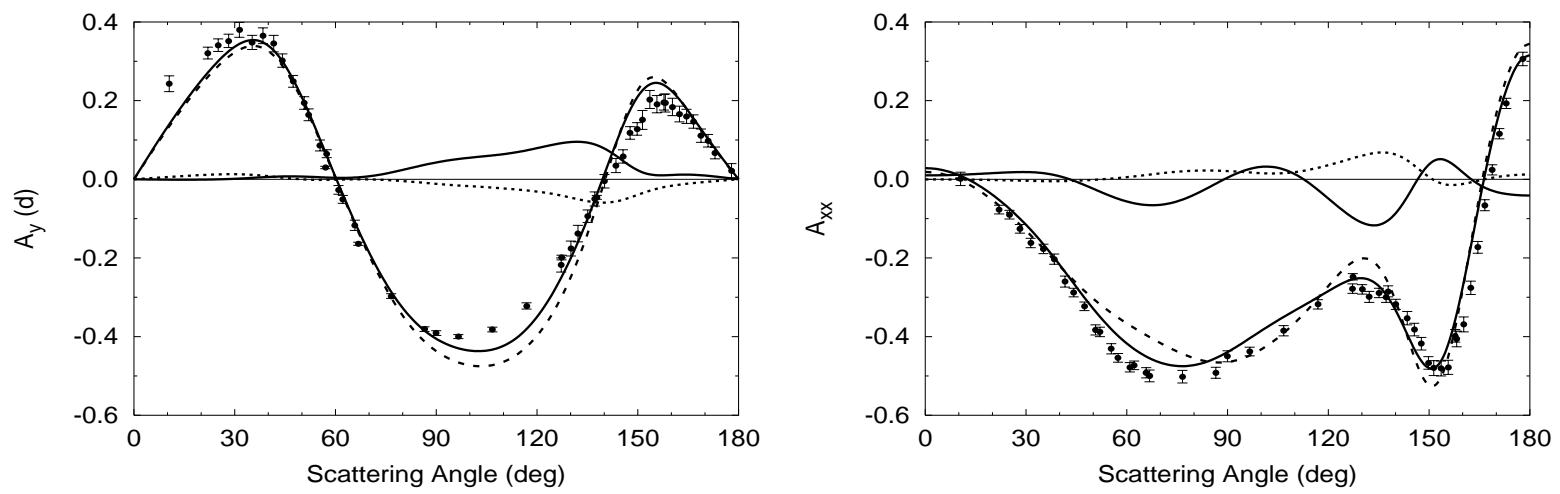

FIG. 11. Deuteron analyzing powers $A_{y}(d)$ and $A_{x x}$ in elastic nucleon-deuteron scattering at $135 \mathrm{MeV}$ nucleon lab energy as function of the c.m. scattering angle. The separated two- and three-nucleon force effects of the $\Delta$ isobar are shown by the two curves around the horizontal zero line. The dotted (solid) curves refer to the effective two-nucleon (three-nucleon) $\Delta$-isobar correction of the observables. The full results with (without) $\Delta$-isobar excitation are also given as solid (dashed) curves for comparison. The experimental data are from Ref. [18] and refer to proton-deuteron scattering. 

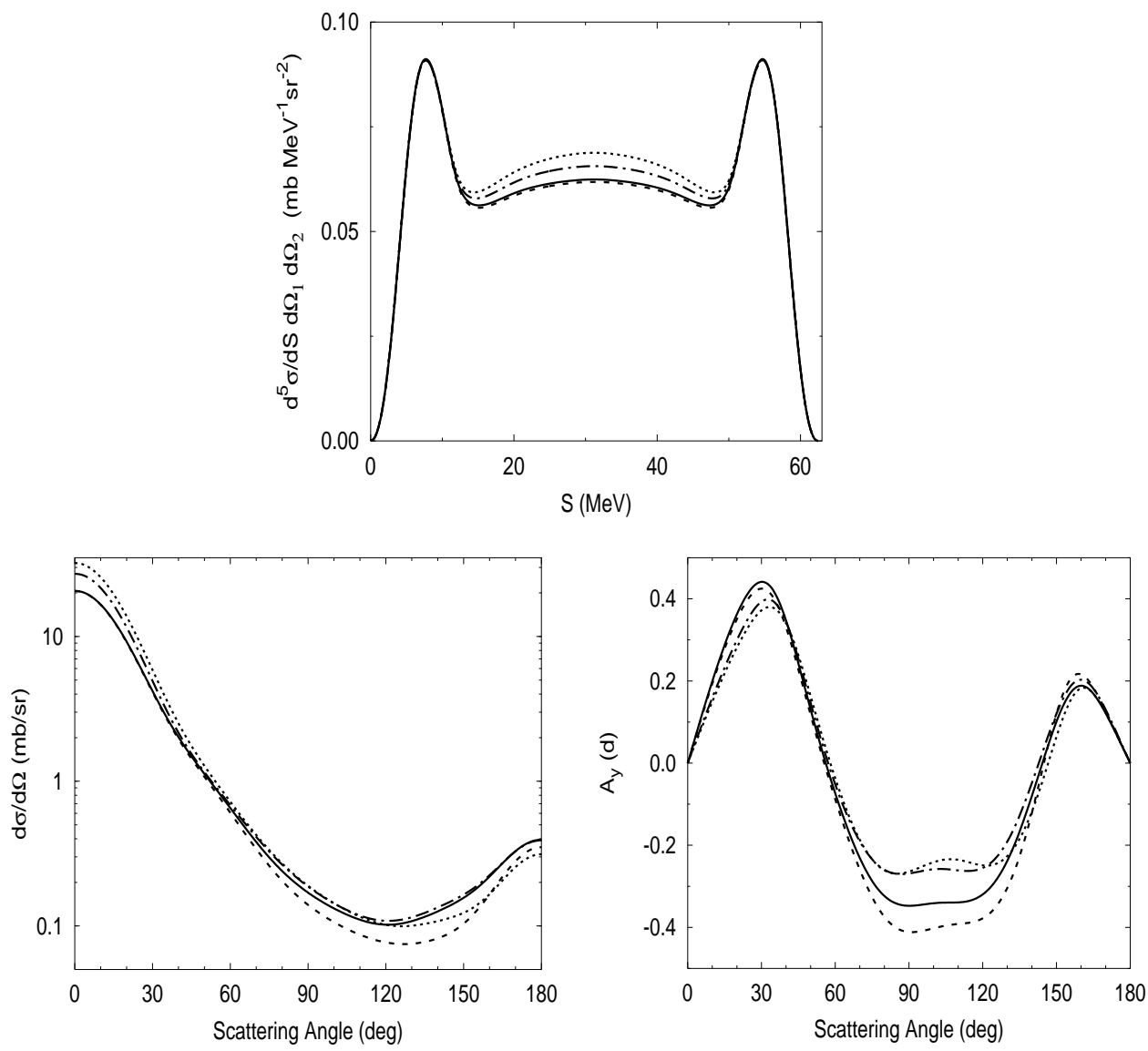

FIG. 12. Differential cross section of nucleon-deuteron breakup at $65 \mathrm{MeV}$ nucleon lab energy as a function of the arclength $S$ along the kinematical curve in the space star configuration $\left(54.0^{\circ}, 54.0^{\circ}, 120.0^{\circ}\right)$ and differential cross section and deuteron analyzing power $A_{y}(d)$ of elastic nucleon-deuteron scattering at $190 \mathrm{MeV}$ nucleon lab energy as functions of the c.m. scattering angle. Compared are predictions of the different coupled-channel potentials, i.e., CD Bonn $+\Delta$ (solid curves), CD Bonn $+\Delta$ (sub1) (dashed-dotted curves), CD Bonn $+\Delta$ (sub2) (dotted curves). Results for the nucleonic CD-Bonn potential without $\Delta$-isobar excitation (dashed curves) are given as reference for the complete $\Delta$-isobar effect.

TABLE III. Retuned $\sigma_{1}, \sigma_{2}$, and $\omega$ parameters in the nucleonic part of the coupled-channel potential. A blank indicates that the boson contribution is not considered. Meson masses $m_{\sigma_{i}}$ and cutoff parameters $\Lambda_{\sigma}=\Lambda_{\sigma_{1}}=\Lambda_{\sigma_{2}}$ and $\Lambda_{\omega}$ are given in MeV; they and $g_{\omega}$ are chosen in a charge-independent form; the coupling constants $g_{\sigma_{i}}$ are charge-dependent. The mass of the $\omega$ meson remains with $781.94 \mathrm{MeV}$ the physical one.

\begin{tabular}{|c|c|c|c|c|c|c|c|c|c|c|c|}
\hline$N N$ & $m_{\sigma_{1}}$ & $m_{\sigma_{2}}$ & $\frac{g_{\sigma_{1}}^{2}(p p)}{4 \pi}$ & $\frac{g_{\sigma_{2}}^{2}(p p)}{4 \pi}$ & $\frac{g_{\sigma_{1}}^{2}(n p)}{4 \pi}$ & $\frac{g_{\sigma_{2}}^{2}(n p)}{4 \pi}$ & $\frac{g_{\sigma_{1}}^{2}(n n)}{4 \pi}$ & $\frac{g_{\sigma_{2}}^{2}(n n)}{4 \pi}$ & $\Lambda_{\sigma}$ & $\frac{g_{\omega}^{2}}{4 \pi}$ & $\Lambda_{\omega}$ \\
\hline${ }^{1} S_{0}$ & 458 & 1225 & 1.92084 & 48.903 & 1.60887 & 55.844 & 1.93591 & 48.847 & 2000 & 20 & 1500 \\
\hline${ }^{3} P_{0}$ & 589 & & 6.2318 & & 6.1004 & & 6.2528 & & 1500 & 20 & 1800 \\
\hline${ }^{3} P_{1}$ & 376 & 1225 & 0.48685 & 0.0 & 0.50895 & 12.337 & 0.49778 & 2.6990 & 1500 & 22 & 2000 \\
\hline${ }^{1} D_{2}$ & 371 & 793 & 0.62970 & 8.8426 & 0.63628 & 8.9343 & 0.64134 & 8.8944 & 2500 & 20 & 1500 \\
\hline${ }^{3} P_{2}$ & 452 & 1225 & 2.8093 & 79.198 & 2.8354 & 78.622 & 2.8218 & 79.035 & 1600 & 20 & 1500 \\
\hline${ }^{3} \mathrm{~F}_{2}$ & 452 & 793 & 1.0511 & 51.447 & 1.1464 & 50.720 & 1.0755 & 51.406 & 1600 & 20 & 1500 \\
\hline${ }^{3} F_{3}$ & 600 & 793 & 6.9214 & 21.972 & 7.2132 & 21.697 & 7.2333 & 20.957 & 2500 & 20 & 1500 \\
\hline${ }^{1} G_{4}$ & 415 & & 1.4793 & & 1.4993 & & 1.5019 & & 2500 & 20 & 1500 \\
\hline${ }^{3} F_{4}$ & 431 & & 2.4451 & & 2.4544 & & 2.4518 & & 2500 & 20 & 1500 \\
\hline${ }^{3} \mathrm{H}_{4}$ & 461 & & 3.5415 & & 3.5977 & & 3.5736 & & 2500 & 20 & 1500 \\
\hline
\end{tabular}


TABLE IV. Meson parameters employed in the potential parts referring to the $\Delta$ isobar. Meson masses $m_{\alpha}$ and cutoff parameters $\Lambda_{\alpha B}$ are given in $\mathrm{MeV}$. All parameters of this table are kept fixed during the fit. The meson masses $m_{\alpha}$ and the other meson parameters $f_{\alpha N N}, g_{\alpha N N}$ and $\Lambda_{\alpha N}$ are taken over from CD Bonn; $g_{\alpha N N}$ and $\Lambda_{\alpha N}$ are identical to $g_{\alpha}$ and $\Lambda_{\alpha}$ of Ref. [9], whereas $f_{\pi N N}$ $=\left(m_{\pi} / 2 m_{N}\right) g_{\pi}$ and $f_{\rho N N}=\left(m_{\rho} / 2 m_{N}\right) g_{\rho}\left(1+f_{\rho} / g_{\rho}\right)$ with $f_{\rho} / g_{\rho}$ $=6.1$ being the ratio of tensor/vector coupling constants of the $\rho$ meson. The coupling strength $f_{\pi N \Delta}$ is taken over from Ref. [1], the others are chosen according to the quark counting rules, i.e., $f_{\rho N \Delta}$ $=f_{\rho N N} f_{\pi N \Delta} / f_{\pi N N}, f_{\alpha \Delta \Delta}=\frac{1}{5} f_{\alpha N N}$, and $g_{\alpha \Delta \Delta}=g_{\alpha N N}$. The cutoff masses $\Lambda_{\alpha \Delta}$ are assumed, they are also not subjected to the fit.

\begin{tabular}{cccccccc}
\hline \hline$\alpha$ & $m_{\alpha}$ & $\frac{f_{\alpha N N}^{2}}{4 \pi}$ & $\frac{g_{\alpha N N}^{2}}{4 \pi}$ & $\Lambda_{\alpha N}$ & $\frac{f_{\alpha N \Delta}^{2}}{4 \pi}$ & $\frac{f_{\alpha \Delta \Delta}^{2}}{4 \pi}$ & $\Lambda_{\alpha \Delta}$ \\
\hline$\pi$ & 138.03 & 0.07348 & 13.6 & 1720 & 0.35 & 0.002939 & 1900 \\
$\rho$ & 769.9 & 7.112 & 0.84 & 1310 & 33.786 & 0.2845 & 1500 \\
$\omega$ & 781.94 & & 20.0 & 1500 & & 1500 & \\
\hline
\end{tabular}

potentials with rather limited phase equivalence. Nevertheless, the resulting $\Delta$-isobar effects seen in the properties of the three-nucleon bound state and in three-nucleon scattering are qualitatively quite similar. The $\Delta$ isobar makes effective two-nucleon contributions as illustrated in Fig. 2 and it mediates an effective three-nucleon force. The effective three-
TABLE V. Retuned $\sigma$ exchange in the direct nucleon- $\Delta$ part of the coupled-channel potential. Note that only the contribution $g_{\sigma N N} g_{\sigma \Delta \Delta} / 4 \pi$ of strength parameters is fitted. The mass $m_{\sigma}$ and the cutoff masses $\Lambda_{\sigma N}$ and $\Lambda_{\sigma \Delta}$ are chosen beforehand and are kept fixed during the fit. The other columns are only shown separately for reasons of correspondence to Table III.

\begin{tabular}{lcccccc}
\hline \hline$N \Delta$ & $m_{\sigma}$ & $\frac{g_{\sigma N N}^{2}}{4 \pi}$ & $\Lambda_{\sigma N}$ & $\frac{g_{\sigma \Delta \Delta}^{2}}{4 \pi}$ & $\Lambda_{\sigma \Delta}$ & $\frac{g_{\sigma N N} g_{\sigma \Delta \Delta}}{4 \pi}$ \\
\hline${ }^{5} D_{0},{ }^{5} P_{3}-{ }^{3} F_{3}-{ }^{5} F_{3}$, & 500 & 5.0 & 1500 & 5.0 & 1500 & 5.0 \\
${ }^{5} D_{4}-{ }^{5} G_{4},{ }^{3} F_{4}-{ }^{5} F_{4}$ & & & & & & \\
${ }^{3} P_{0},{ }^{3} P_{1}-{ }^{5} P_{1}-{ }^{5} F_{1}$, & 500 & 0.0 & 1500 & 0.0 & 1500 & 0.0 \\
${ }^{3} P_{2}-{ }^{5} P_{2}$ & 500 & 8.7 & 1500 & 8.7 & 1500 & 8.7 \\
${ }^{5} S_{2}-{ }^{5} D_{2}-{ }^{5} G_{2}$ & 500 & \\
\hline \hline
\end{tabular}

nucleon force simulates the two-pion exchange FujitaMiyazawa force [28] and the three-pion ring parts of the Illinois forces [29] in a reducible energy-dependent form. In contrast to those irreducible three-nucleon forces based solely on $\pi$ exchange, the effective three-nucleon force arising from the coupled-channel potential takes $\pi, \rho, \omega$, and $\sigma$ exchanges into account. The coupled-channel potential makes all contributions to the three-nucleon force mutually consistent.

Despite $\Delta$-isobar effects the coupled-channel potential re-
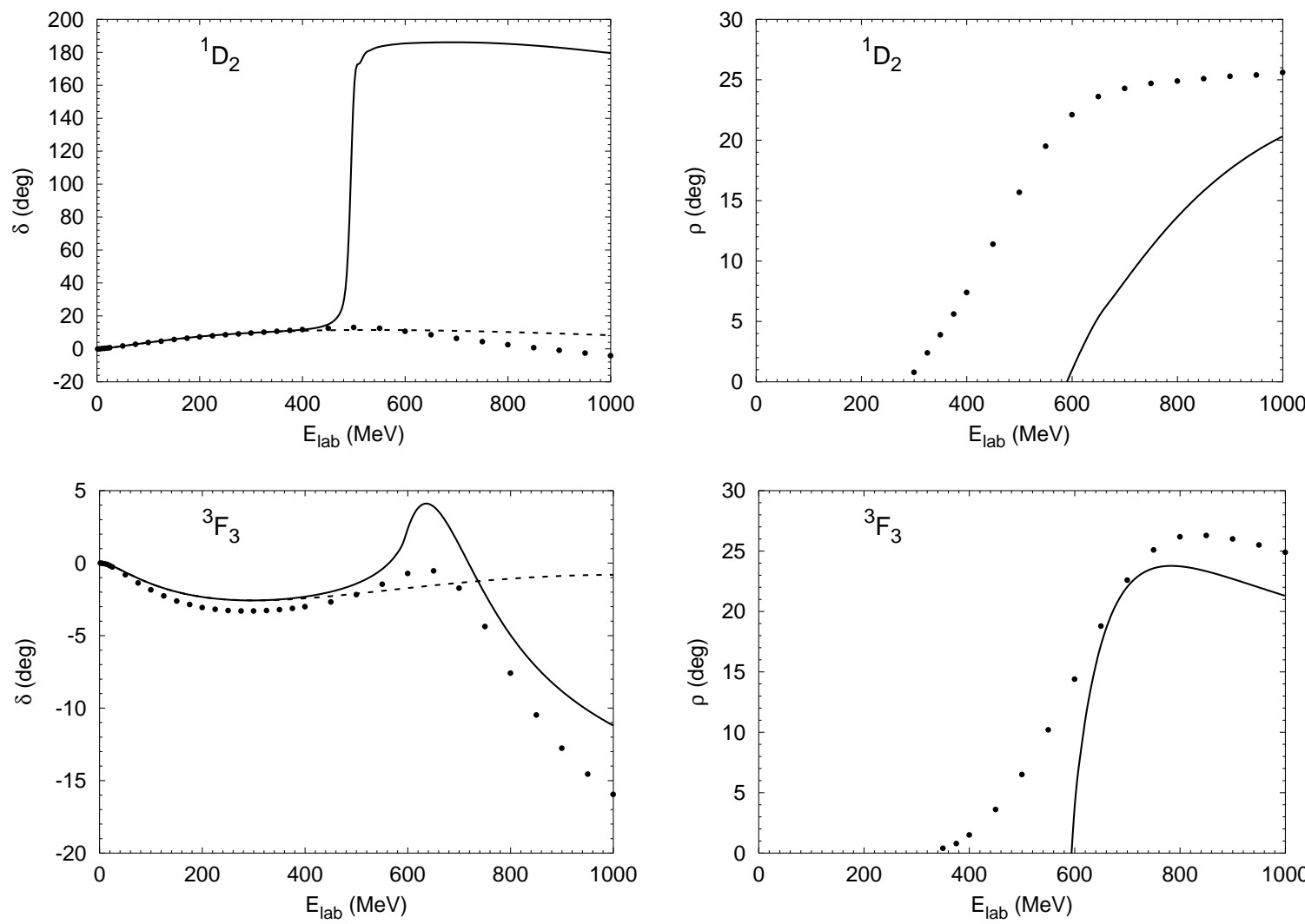

FIG. 13. ${ }^{1} D_{2}$ and ${ }^{3} F_{3} n p$ phase shifts $\delta$ and inelasticities $\rho$ as functions of nucleon lab energy. Results of the coupled-channel potential with $\Delta$-isobar excitation (solid curves) are compared with results of the CD-Bonn potential (dashed curves), for which the inelasticity $\rho$ is exactly zero. The experimental data are from Ref. [30]; in the fit of this paper only the phase shifts below $350 \mathrm{MeV}$ nucleon lab energy are considered. 
mains unable to account for three-nucleon binding in full. Thus, the addition of an irreducible three-nucleon force, from which $\Delta$-isobar contributions are removed, is required, in order to at least cure that binding shortcoming of our dynamic input for the description of three-nucleon scattering. This task could not be done yet. With respect to threenucleon scattering, $\Delta$-isobar effects become more visible at higher energies; they are often beneficial for a satisfactory description, though their success is not a general one for all measured observables.

\section{ACKNOWLEDGMENTS}

The authors thank K. Ermisch, N. Kalantar-Nayestanaki, and K. Sekiguchi for a discussion of the new experimental data used in this paper for a comparison with theoretical predictions. A. D. acknowledges a valuable DAAD grant for graduate studies at the University of Hannover. The work of R. M. is supported by the U.S. National Science Foundation under Grant No. PHY-0099444. The numerical calculations were performed at Regionales Rechenzentrum für Niedersachsen.

\section{APPENDIX: PARAMETERS AND SPECIAL PROPERTIES OF THE COUPLED-CHANNEL POTENTIAL}

The force parameters of the fitted coupled-channel potential are collected in this appendix. They are quoted with different accuracies. This fact is standard: The fit determines some parameters more sensitively than others. For reasons of reproducibility, we give the parameters with all digits, used in the practical calculations.
Table III collects the retuned parameters of the nucleonic part of the coupled-channel potential; the retuning is done for the $\sigma_{1}, \sigma_{2}$ and, in ${ }^{3} P_{0}$ and ${ }^{3} P_{1}$ partial waves, also for the $\omega$ exchange. The $\sigma$ exchange is phenomenological; CD Bonn uses it in a partial-wave-dependent form; its retuning is well justified. The retuning of the $\omega$ exchange is only minor; nevertheless, the retuned $\sigma_{1}, \sigma_{2}$, and $\omega$ exchanges have more partial-wave dependence than the underlying CD-Bonn potential. The force parameters determining the potential parts connected with the $\Delta$ isobar are given in Tables IV and V. Table IV contains all parameters chosen, before the coupledchannel potential is subjected to the fit. Table V contains the only fit parameter, i.e., $g_{\sigma N N} g_{\sigma \Delta \Delta} / 4 \pi$; it refers exclusively to $\sigma$ meson exchange.

The coupled-channel potential has an inelastic channel, the nucleon- $\Delta$ channel. Thus, the potential yields inelasticities; they are not realistic, since they are not pionic. Nevertheless, for reasons of curiosity, Fig. 13 shows the resulting ${ }^{1} D_{2}$ and ${ }^{3} F_{3}$ phase shifts and inelasticities also in the energy domain of scattering beyond the pion-production threshold not used for the fit. As expected, the produced inelasticities do not account for data. First, due to the neglect of the coupling to pion states the inelastic threshold is wrong. Second, the ${ }^{1} D_{2}$ nucleon-nucleon channel is coupled to the ${ }^{5} S_{2}$ nucleon- $\Delta$ channel; without that coupling the nucleon- $\Delta$ potential supports a bound state; this is the reason for the sharp phase shift increase through $\pi / 2$ which the coupling to pion states would efficiently smear out. In contrast to ${ }^{1} D_{2}$, the predictions for the ${ }^{3} F_{3}$ phase shifts show some realistic features qualitatively. In the energy domain of inelasticity the nucleonic CD-Bonn potential is also unrealistic.
[1] C. Hajduk, P.U. Sauer, and W. Strueve, Nucl. Phys. A405, 581 (1983).

[2] S. Nemoto, K. Chmielewski, J. Haidenbauer, U. Meyer, S. Oryu, and P.U. Sauer, Few-Body Syst. 24, 241 (1998).

[3] S. Nemoto, K. Chmielewski, S. Oryu, and P.U. Sauer, Phys. Rev. C 58, 2599 (1998).

[4] K. Chmielewski, A. Deltuva, A.C. Fonseca, S. Nemoto, and P.U. Sauer, Phys. Rev. C 67, 014002 (2003).

[5] A. Deltuva, K. Chmielewski, and P.U. Sauer, Phys. Rev. C 67, 034001 (2003).

[6] L.P. Yuan, K. Chmielewski, M. Oelsner, P.U. Sauer, A.C. Fonseca, and J. Adam, Jr., Few-Body Syst. 32, 83 (2002).

[7] H. Pöpping, P.U. Sauer, and X.-Z. Zhang, Nucl. Phys. A474, 557 (1987); A550, 563(E) (1992).

[8] P.U. Sauer, Prog. Part. Nucl. Phys. 16, 35 (1986).

[9] R. Machleidt, Phys. Rev. C 63, 024001 (2001).

[10] V.G.J. Stoks, R.A.M. Klomp, C.P.F. Terheggen, and J.J. de Swart, Phys. Rev. C 49, 2950 (1994).

[11] R.B. Wiringa, V.G.J. Stoks, and R. Schiavilla, Phys. Rev. C 51, 38 (1995).

[12] A. Picklesimer, R.A. Rice, and R. Brandenburg, Phys. Rev. C 44, 1359 (1991).

[13] V. Stoks and J.J. de Swart, Phys. Rev. C 47, 761 (1993).

[14] C.R. Howell et al., Phys. Lett. B 444, 252 (1998).
[15] M.T. Peña, H. Henning, and P.U. Sauer, Phys. Rev. C 42, 855 (1990).

[16] G. Derrick and J.M. Blatt, Nucl. Phys. 8, 310 (1958).

[17] E.P. Harper, Y.E. Kim, and A. Tubis, Phys. Rev. C 6, 126 (1972).

[18] K. Sekiguchi et al., Phys. Rev. C 65, 034003 (2002).

[19] H. Posma and R. Wilson, Phys. Rev. 121, 1229 (1961).

[20] R.E. Adelberger and C.N. Brown, Phys. Rev. D 5, 2139 (1972).

[21] K. Ermisch, Ph.D. thesis, University of Groningen, 2003.

[22] K. Ermisch et al., Phys. Rev. Lett. 86, 5862 (2001).

[23] R. Bieber et al., Phys. Rev. Lett. 84, 606 (2000).

[24] K. Sekiguchi, Ph.D. thesis, University of Tokyo, 2001.

[25] M. Allet et al., Phys. Rev. C 50, 602 (1994).

[26] J. Kuros-Zolnierczuk, H. Witala, J. Golak, H. Kamada, A. Nogga, R. Skibinski, and W. Glöckle, Phys. Rev. C 66, 024003 (2002).

[27] A. Deltuva, K. Chmielewski, and P.U. Sauer, Phys. Rev. C 67, 054004 (2003).

[28] J. Fujita and H. Miyazawa, Prog. Theor. Phys. 17, 360 (1957).

[29] S.C. Pieper, V.R. Pandharipande, R.B. Wiringa, and J. Carlson, Phys. Rev. C 64, 014001 (2001).

[30] R.A. Arndt, L.D. Roper, R.A. Bryan, R.B. Clark, B.J. VerWest, and P. Signell, Phys. Rev. D 28, 97 (1983). 